\title{
New quantum transition in Weyl semimetals with correlated disorder
}

\author{
T. Louvet, D. Carpentier, and A. A. Fedorenko \\ Univ Lyon, Ens de Lyon, Univ Claude Bernard, CNRS, Laboratoire de Physique, F-69342 Lyon, France
}

(Dated: August 6, 2018)

\begin{abstract}
A Weyl semimetal denotes an electronic phase of solids in which two bands cross linearly. In this paper we study the effect of a spatially correlated disorder on such a phase. Using a renormalization group analysis, we show that in three dimensions, three scenarios are possible depending on the disorder correlations. A standard transition is recovered for short range correlations. For disorder decaying slower than $1 / r^{2}$, the Weyl semimetal is unstable to any weak disorder and no transition persists. In between, a new phase transition occurs. This transition still separates a disordered metal from a semi-metal, but with a new critical behavior that we analyze to two-loop order.
\end{abstract}

\section{INTRODUCTION}

The Anderson localization transition is the seminal exemple of a disorder driven quantum phase transition between a metallic and an insulating phase [1]. Recently, a different transition has attracted a lot of attention, in which disorder drives a zero gap semi-conductor with linear band crossing into a diffusive metal (for review see [2]).

In this second case, the density of state (DOS) at the band crossing is actually increased by the disorder, as opposed to the standard situation for the Anderson transition. Interest in these phases which we will call semimetals has been steadily developing for several years, fueled by intense activity on both theoretical and experimental aspects. It is now well known that relativistic massless fermions emerge as 2D electronic excitations in graphene and as surface states of 3D topological insulators. Two years ago, the successive discovery of materials hosting linear band crossings in 3D has revived the excitement around the field [3, 4]. Indeed, the 3D case is special in that a linear crossing between two bands, called a Weyl point, possesses a topological property which encodes its protection against gap opening by perturbations preserving translational invariance. Quite naturally, a weak scalar disorder, which is inescapably present in real materials, is expected to preserve the band crossing of these phases.

Such a stability with respect to disorder can be cast into the renormalization group ( $\mathrm{RG}$ ) framework. While in two dimensions any amount of disorder is relevant and destabilizes the band crossing, a weak disorder is irrelevant for three dimensional fermions with linear dispersion relation. The crossing band point persists in the presence of weak quenched disorder, which results only in a non-universal renormalization of the Fermi velocity. Thus, a weakly disordered system behaves qualitatively as a clean sample: the density of states (DOS) vanishes quadratically with energy, up to exponentially small corrections due to rare events [5, 6. It will also feature pseudoballistic transport properties [7] and a vanishing zerofrequency optical conductivity [8]. However, a strong enough disorder drives the system from a semimetal to a disordered metallic phase through a transition which is different from the Anderson one. This disordered metal is characterized by a finite DOS at zero energy 9, but its precise nature, e.g. Anderson insulator or diffusive metal, depends on the precise nature of the phase, e.g. Dirac versus Weyl semimetals or number of cones [10. 14. This transition has been recently intensively studied both numerically [15-18] as well as analytically using $2+\varepsilon$ [19,20] and $4-\varepsilon$ 21, 22, expansions. In particular, the transition is characterized through the critical exponents $\nu$ and $z$ : at this transition, the correlation length diverges as $\xi \sim\left|\Delta-\Delta_{c}\right|^{-\nu}$ where $\Delta_{c}$ is the critical disorder strength, while the dynamic critical exponent $z$ is defined trough the scaling between energy and momenta at the transition $\omega \sim k^{z}$. Besides, it was found that exactly at the transition the wave functions and local DOS exhibit multifractality similar to the Anderson transition but with different universality classes [21, 23].

It is well known that correlations of the disorder potential, overlooked in this previous studies, can be present experimentally and change the nature of the transition. This is indeed the case for the Anderson transition [24]. Moreover, the low energy properties of the Dirac phase in graphene are known to be sensitive to disorder correlations 25]. Such correlations may originate from the presence of linear dislocations, planar grain boundaries, unscreened charge impurities, etc.

In the present paper we consider the effects of longrange disorder correlations on the semimetal-diffusive metal transition in the Weyl semimetals restricting consideration to a single cone.

The paper is organized as follows. Section II introduces the model which is renormalized in Sec. III. We study the phase diagram and calculate the critical exponents to two-loop order in Sec. IV and conclude in Sec. V.

\section{MODEL}

Relativistic fermions moving in a $d$-dimensional space in the presence of an external potential $V(r)$ can be described by the euclidian action

$$
S=\int d^{d} r \int d \tau \bar{\psi}(\mathbf{r}, \tau)\left(\partial_{\tau}-i \gamma_{j} \partial^{j}+V(\mathbf{r})\right) \psi(\mathbf{r}, \tau)
$$


where $\psi$ and $\bar{\psi}$ are independent Grassmann fields and $\tau$ is the imaginary time. The three-dimensional (3D) Weyl fermions corresponds to $\gamma_{j}=\sigma_{j}, j=1,2,3$ given by the Pauli matrices. In general the $\gamma_{j}$ are elements of a Clifford algebra satisfying the anticommutation relations: $\gamma_{i} \gamma_{j}+\gamma_{j} \gamma_{i}=2 \delta_{i j} \mathbb{I}$, and $i, j=1, \ldots, d$. We assume that the disorder potential $V(\mathbf{r})$ is a random Gaussian variable with zero mean and the variance $\overline{V(\mathbf{r}) V\left(\mathbf{r}^{\prime}\right)}=g\left(\mathbf{r}-\mathbf{r}^{\prime}\right)$, $g(r) \sim r^{-a}$. For convenience, we fix the normalization of the variance in the Fourier space

$$
\tilde{g}(k)=\Delta_{1}+\Delta_{2} k^{a-d},
$$

which must be positive. The $\Delta_{1}$ term in Eq. (2) corresponds to the short-range (SR) disorder since it becomes the Dirac $\delta$-function in real space: the impact of such uncorrelated disorder has been studied previously in Refs. [15 22]. The strength of the long-range (LR) correlated disorder is given by $\Delta_{2}$. To average over disorder we use the replica trick and introduce $N$ copies of the original system. After averaging over disorder we arrive at the replicated effective action

$$
\begin{aligned}
S_{\mathrm{eff}}= & \int d \tau d^{d} r \bar{\psi}_{\alpha}(\mathbf{r}, \tau)\left(\partial_{\tau}-i \gamma_{j} \partial^{j}\right) \psi_{\alpha}(\mathbf{r}, \tau) \\
& -\frac{1}{2} \int d \tau_{1} d \tau_{2} d^{d} r_{1} d^{d} r_{2} g\left(\mathbf{r}_{1}-\mathbf{r}_{2}\right) \\
& \times \bar{\psi}_{\alpha}\left(\mathbf{r}, \tau_{1}\right) \psi_{\alpha}\left(\mathbf{r}, \tau_{1}\right) \bar{\psi}_{\beta}\left(\mathbf{r}, \tau_{2}\right) \psi_{\beta}\left(\mathbf{r}, \tau_{2}\right)
\end{aligned}
$$

where summation over repeated replica indices $\alpha, \beta=$ $1, \ldots, N$ is implied. The properties of the original system with quenched disorder can be obtained by taking the limit $N \rightarrow 0$. Introducing the Matsubara frequency $\omega$ the action (3) can be rewritten in the Fourier space as

$$
\begin{aligned}
S & =\int_{k, \omega} \bar{\psi}_{\alpha}(-\mathbf{k},-\omega)(\gamma \mathbf{k}-i \omega) \psi_{\alpha}(\mathbf{k}, \omega) \\
& -\frac{1}{2} \int_{k_{i}, \omega_{i}} \tilde{g}\left(\mathbf{k}_{1}+\mathbf{k}_{2}\right) \bar{\psi}_{\alpha}\left(\mathbf{k}_{1},-\omega_{1}\right) \psi_{\alpha}\left(\mathbf{k}_{2}, \omega_{1}\right) \\
& \times \bar{\psi}_{\beta}\left(\mathbf{k}_{3},-\omega_{2}\right) \psi_{\beta}\left(-\mathbf{k}_{1}-\mathbf{k}_{2}-\mathbf{k}_{3}, \omega_{2}\right)
\end{aligned}
$$

where $\int_{k}:=\int \frac{d^{d} k}{(2 \pi)^{d}}$ and $\int_{\omega}:=\int \frac{d \omega}{(2 \pi)}$. We now define the correlation functions with insertions of the composite operator $\mathcal{O}(r):=\bar{\psi}_{\alpha}(r) \psi_{\alpha}(r)$,

$$
\begin{aligned}
& G^{(2 n, l)}(\{x\},\{y\},\{z\}) \\
& =\left\langle\bar{\psi}_{\alpha_{1}}\left(x_{1}\right) \psi_{\alpha_{1}}\left(y_{1}\right) \ldots \bar{\psi}_{\alpha_{n}}\left(x_{n}\right) \psi_{\alpha_{n}}\left(y_{n}\right) \mathcal{O}\left(z_{1}\right) \ldots \mathcal{O}\left(z_{l}\right)\right\rangle
\end{aligned}
$$

where we used the shortcut notation $x_{i}:=\left(r_{i}, \tau_{i}\right)$. For example, the local DOS can be found from the retarded Green function

$$
\rho(E)=-\frac{1}{\pi} \operatorname{Im} G^{R}(r, r, E)
$$

which is related to $G^{(0,1)}(r, \omega)$ by the analytic continuation $i \omega \rightarrow E+i 0$. The correlation functions (5) can be calculated perturbatively in small $\Delta_{1}$ and $\Delta_{2}$. Each term of this perturbation series can be represented as a Feynman diagram in which lines stand for the the bare propagator

$$
\left\langle\bar{\psi}_{\alpha}(\mathbf{k}, \omega) \psi_{\beta}(-\mathbf{k},-\omega)\right\rangle_{0}=\delta_{\alpha \beta} \frac{\gamma \mathbf{k}+i \omega}{k^{2}+\omega^{2}} .
$$

and there are two types of vertices which correspond to $\Delta_{1}$ and $\Delta_{2}$ terms in action (4) with $\tilde{g}(k)$ given by Eq. (2). Both of them transmit only momenta but not frequency and the second vertex explicitly depends on the transmitted momenta as $k^{a-d}$.

\section{RENORMALIZATION AND SCALING BEHAVIOR}

Dimensional analysis shows that weak disorder is irrelevant for $d>2$, nevertheless the system can undergo a phase transition to a diffusive metal for strong enough disorder. The correlation functions (5) computed perturbatively in small disorder turn out to be diverging in $d=2$ which is the lower critical dimension of the transition. To describe the scaling behavior of the system in the vicinity of this transition we apply the field-theoretic renormalization group to two-loop order. To that end we calculate the correlation functions using dimensional regularization. Following Ref. [26] we perform a double expansion in $d=2+\varepsilon$ and $a=2+\delta$ in such a way that the UV divergences are converted into the poles in $\varepsilon$ and $\delta$. In the framework of the minimal subtraction scheme we consider ratios like $\varepsilon / \delta$ to be finite in the limit $\varepsilon, \delta \rightarrow 0$. We do not include them into the counterterms, choosing the latter to be the pole part only. The poles in $\varepsilon$ and $\delta$ can be accumulated in the renormalization factors: $Z_{\psi}$, $Z_{\omega}, Z_{1}$ and $Z_{2}$, so that the renormalized action can be written as

$$
\begin{aligned}
& S_{R}=\int_{k, \omega} \bar{\psi}_{\alpha}\left(Z_{\psi} \gamma \mathbf{k}-i Z_{\omega} \omega\right) \psi_{\alpha} \\
& -\frac{1}{2} \int_{k_{i}, \omega_{i}}\left(\mu^{-\varepsilon} Z_{1} \Delta_{1}+\mu^{-\delta} Z_{2} \Delta_{2}|\mathbf{k}|^{a-d}\right) \bar{\psi}_{\alpha} \psi_{\alpha} \bar{\psi}_{\beta} \psi_{\beta} .
\end{aligned}
$$

Here we have introduced the renormalized fermionic fields $\psi, \bar{\psi}$ and the renormalized dimensionless coupling constants $\Delta_{1}$ and $\Delta_{2}$ on the mass scale $\mu$. In what follows we will denote the bare variables by a ring. The renormalized variables are related to the bare ones by

$$
\begin{aligned}
& \stackrel{\circ}{\psi}=Z_{\psi}^{1 / 2} \psi, \quad \stackrel{\circ}{\psi}=Z_{\psi}^{1 / 2} \bar{\psi}, \\
& \stackrel{\circ}{j}_{j}=Z_{\omega} Z_{\psi}^{-1} \omega_{j}, \quad \stackrel{\circ}{O}=Z_{\omega} Z_{\psi}^{-1} O, \\
& \stackrel{\circ}{\Delta}_{1}=\frac{2 \mu^{-\varepsilon}}{K_{d}} \frac{Z_{1}}{Z_{\psi}^{2}} \Delta_{1}, \quad \stackrel{\circ}{\Delta}_{2}=\frac{2 \mu^{-\delta}}{K_{d}} \frac{Z_{2}}{Z_{\psi}^{2}} \Delta_{2} .
\end{aligned}
$$

For the sake of simplicity we have also included $K_{d} / 2$ into definition of the renormalized coupling constants in 
Eqs. 111. $K_{d}=2 \pi^{d / 2} /\left((2 \pi)^{d} \Gamma(d / 2)\right)$ is the surface area of the $d$-dimensional unite sphere divided by $(2 \pi)^{d}$ coming from the angular integration in the Feynmann diagrams. The renormalized and the bare Green functions are related by

$$
\dot{\circ}^{(2 n, l)}(\{p, \stackrel{\circ}{\omega}\}, \stackrel{\circ}{\Delta})=Z_{\omega}^{l} Z_{\psi}^{n-l} G^{(2 n, l)}(\{p, \omega\}, \Delta, \mu),
$$

where $\Delta:=\left\{\Delta_{1}, \Delta_{2}\right\}$. Using that the bare Green functions $\stackrel{G}{G}^{(2 n, l)}$ do not depend on the renormalization scale $\mu$ one can derive the $\mathrm{RG}$ flow equation

$$
\begin{aligned}
& {\left[\sum_{j} p_{j} \frac{\partial}{\partial p_{j}}+(1+\gamma(\Delta)) \sum_{j} \omega_{j} \frac{\partial}{\partial \omega_{j}}\right.} \\
& \quad+\sum_{i=1,2} \beta_{i}(\Delta) \frac{\partial}{\partial \Delta_{i}}+d(2 n-1)-n\left(d-1+\eta_{\psi}(\Delta)\right) \\
& \quad+l(1+\gamma(\Delta))] G^{(2 n, l)}(\{p, \omega\}, \Delta, \mu)=0,
\end{aligned}
$$

where we have defined the scaling functions

$$
\begin{aligned}
& \beta_{i}(\Delta)=-\left.\mu \frac{\partial \Delta_{i}}{\partial \mu}\right|_{\delta}, \\
& \eta_{\psi}(\Delta)=-\sum_{i=1,2} \beta_{i}(\Delta) \frac{\partial \ln Z_{\psi}}{\partial \Delta_{i}} \\
& \eta_{\omega}(\Delta)=-\sum_{i=1,2} \beta_{i}(\Delta) \frac{\partial \ln Z_{\omega}}{\partial \Delta_{i}} \\
& \gamma(\Delta)=\eta_{\omega}(\Delta)-\eta_{\psi}(\Delta) .
\end{aligned}
$$

The solutions of Eq. (13) can be found by using the method of characteristics. The characteristics are lines in the space of $p_{i}, \Delta, \omega_{i}$ along which Eq. 13 can be rewritten as an ordinary differential equation of the first order. The characteristics lines can be parameterized by the auxiliary parameter $\xi$ which will be later identified with the correlation length. The lines are given by equations:

$$
\begin{aligned}
& \frac{d p_{j}(\xi)}{d \ln \xi}=p_{j}(\xi), \\
& \frac{d \Delta_{i}(\xi)}{d \ln \xi}=\beta_{i}(\Delta(\xi)), \\
& \frac{d \omega_{j}(\xi)}{d \ln \xi}=[1+\gamma(\Delta(\xi))] \omega_{j}(\xi),
\end{aligned}
$$

with the initial conditions $\Delta(1)=\Delta, p_{j}(1)=p_{j}$, and $\omega_{j}(1)=\omega_{j}$. The solution of Eq. 13p propagates along the characteristics lines according to the ordinary differential equation equation

$$
\begin{aligned}
\frac{d \ln H_{2 n, l}(\xi)}{d \ln \xi}= & d(2 n-1)-n\left(d-1+\eta_{\psi}(\Delta(\xi))\right) \\
& +l(1+\gamma(\Delta)) .
\end{aligned}
$$

with the initial conditions $H_{2 n, l}(1)=1$. Thus the solution of Eq. 13) satisfies

$$
G^{(2 n, l)}\left(p_{j}, \omega_{j}, \Delta\right)=H_{2 n, l}(\xi) G^{(2 n, l)}\left(p_{j}(\xi), \omega_{j}(\xi), \Delta(\xi)\right) .
$$

Since the DOS is related to $G^{(0,1)}$ by Eq. (6) it obeys the scaling relation

$$
\rho(\omega, \Delta)=H_{0,1}(\xi) \rho(\omega(\xi), \Delta(\xi)) .
$$

Let us now assume that the RG flow (19) has a fixed point $(\mathrm{FP}) \Delta^{*}=\left(\Delta_{1}^{*}, \Delta_{2}^{*}\right)$ defined as

$$
\beta_{i}\left(\Delta^{*}\right)=0, \quad i=1,2 .
$$

To determine the stability properties of the FP one can linearize the flow equation in its vicinity computing the stability matrix

$$
\mathcal{M}_{i j}=\left.\frac{\partial \beta_{i}(\Delta)}{\partial \Delta_{j}}\right|_{\Delta^{*}}
$$

which has two eigenvalues $\lambda_{1}$ and $\lambda_{2}$. The positive eigenvalues of the matrix (25) correspond to the unstable directions in the plane $\left(\Delta_{1}, \Delta_{2}\right)$. The transition if it exists is controlled by a FP which has only one of two directions unstable, e.g. $\lambda_{1}>0$ and $\lambda_{2}<0$. Introducing the eigenvector $\Delta-\Delta^{*}$ associated with $\lambda_{1}>0$ the scaling formula 22 in the vicinity of the FP (24) can be written as

$$
\begin{aligned}
& G^{(2 n, l)}\left(p_{i}, \omega_{i}, \Delta\right) \\
& \quad=\xi^{d(2 n-1)-2 n d_{\psi}+l z} g_{2 n, l}\left(p_{i} \xi, \omega_{i} \xi^{z},\left|\Delta-\Delta^{*}\right| \xi^{1 / \nu}\right) .
\end{aligned}
$$

Here $\left|\Delta-\Delta^{*}\right|$ is the length of the eigenvector and we have defined the critical exponents for the correlation length $\xi$,

$$
\xi \sim\left|\Delta-\Delta^{*}\right|^{-\nu}, \quad \frac{1}{\nu}=\lambda_{1},
$$

the dynamic critical exponent

$$
\omega \sim k^{z}, \quad z=1+\gamma\left(\Delta^{*}\right)
$$

and the anomalous dimension of the fields $\psi$ and $\bar{\psi}$

$$
d_{\psi}=\frac{1}{2}\left[d-1+\eta_{\psi}\left(\Delta^{*}\right)\right] .
$$

For instance, the two-point correlation function, which gives the momentum distribution at the transition, behaves as

$$
G^{(2,0)}(p) \sim p^{-1+\eta_{\psi}\left(\Delta^{*}\right)}, \quad G^{(2,0)}(r) \sim \frac{1}{r^{d-1+\eta_{\psi}\left(\Delta^{*}\right)}} .
$$

The scaling formula 23 for the DOS in the vicinity of the FP 24) has the form

$$
\rho(\omega)=\xi^{z-d} \rho_{0}\left(\omega \xi^{z},\left|\Delta-\Delta^{*}\right| \xi^{1 / \nu}\right) .
$$




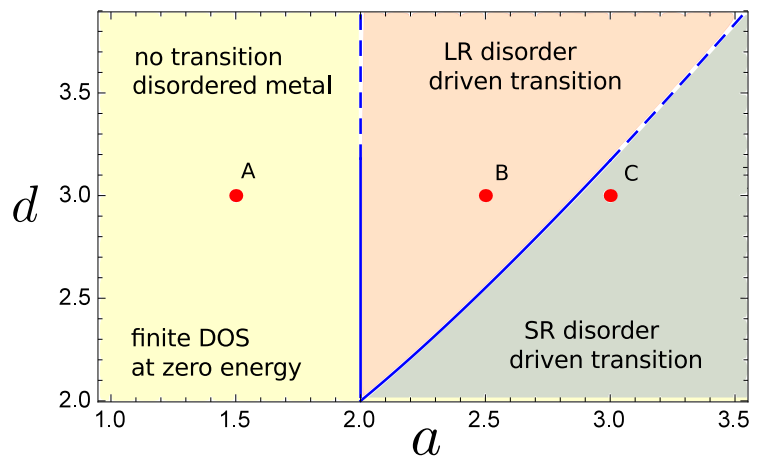

FIG. 1. Domains of existence of the transition as a function of $a$ and $d$ : (i) in the left region there is no transition, the disorder is always relevant and the disordered metal is the only phase. The typical RG flow computed at the point $\mathrm{A}$ $[a=3 / 2, d=3]$ is shown in Fig. 2, in the middle region there is a new transition from a semimetal phase to a metallic phase controlled by the LR FP. The typical RG flow computed at the point $\mathrm{B}[a=5 / 2, d=3]$ is shown in Fig. 3 in the right region there is a transition from a semimetal phase to a metallic phase controlled by the SR FP. The typical RG flow computed at the point $\mathrm{C}[a=3, d=3]$ is shown in Fig. 4

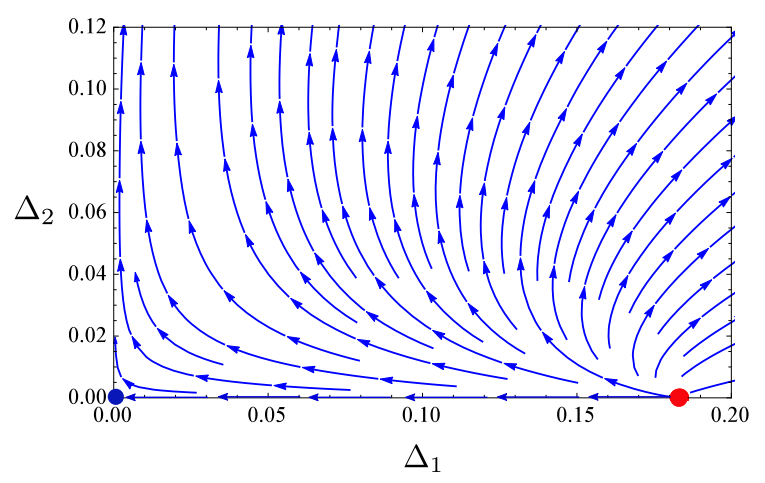

FIG. 2. Strongly disordered phase: runaway of the RG flow for $d=3(\varepsilon=1)$ and $a=3 / 2(\delta=-1 / 2)$ corresponding to the point A in Fig. 1. The red dot is the SR FP which is fully unstable. The blue dot is the Gaussian FP corresponding to the semimetal phase.

\section{TRANSITIONS: EXISTENCE AND CRITICAL BEHAVIOR}

Using the two-loop diagrams computed in Ref. [26] we derive the beta functions (14):

$$
\begin{aligned}
\beta_{1}\left(\Delta_{1}, \Delta_{2}\right)= & -\varepsilon \Delta_{1}+4 \Delta_{1}^{2}+4 \Delta_{1} \Delta_{2}+8 \Delta_{1}^{3} \\
& +20 \Delta_{1}^{2} \Delta_{2}+4 \Delta_{2}^{3}+16 \Delta_{1} \Delta_{2}^{2} \\
\beta_{2}\left(\Delta_{1}, \Delta_{2}\right)= & -\delta \Delta_{2}+4 \Delta_{2}^{2}+4 \Delta_{1} \Delta_{2}+4 \Delta_{2}^{3} \\
& +4 \Delta_{1}^{2} \Delta_{2}+8 \Delta_{1} \Delta_{2}^{2}
\end{aligned}
$$

the dynamic critical exponent 28:

$$
z\left(\Delta_{1}, \Delta_{2}\right)=1+2\left(\Delta_{1}+\Delta_{2}\right)+2\left(\Delta_{1}+\Delta_{2}\right)^{2},
$$

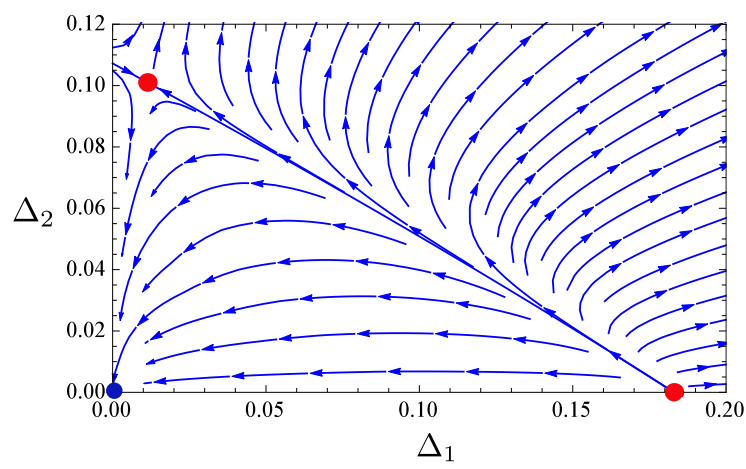

FIG. 3. New LR transition: the RG flow for $d=3(\varepsilon=1)$ and $a=5 / 2(\delta=1 / 2)$ corresponding to the point B in Fig. 1 . The right red dot is the SR FP which is fully unstable. The left red dot is the new LR FP which controls the transition between a semimetal and a disordered metal phase and which gives rise to a new universality class different from the SR case. The blue dot is the Gaussian FP corresponding to the semimetal phase.

and the anomalous dimension (15) of the fermionic fields

$$
\eta_{\psi}\left(\Delta_{1}, \Delta_{2}\right)=-2 \Delta_{1}^{2}+2 \Delta_{2}^{2}-\frac{4 \varepsilon}{\delta} \Delta_{2}\left(\Delta_{1}+\Delta_{2}\right) .
$$

Note that the ratio $\varepsilon / \delta$ in Eq. (35) is finite in our regularization scheme.

We now analyze the RG flow derived from Eqs. (32)(35) that is summarized in Figures 1. 4. We are interested in the effect of an additional LR correlated disorder on the SR disorder driven transition: our results on the stability of the SR fixed point with respect to additional $\Delta_{2}$ distinguish between three different domains, as shown on Fig. 1. First note that the case $d=2(\varepsilon=0)$, corresponding to graphene, is special since it corresponds to the lower critical dimension where no transition occurs: SR disorder is marginally relevant and drives the system to a strong disordered metallic phase, characterized by a finite zero-energy DOS 25. For dimension $d$ greater than 2 we must distinguish three regimes of disorder correlations :

(A) For $a<2(\delta<0)$ i.e. when the long-range disorder correlations decay slower than $1 / r^{2}$, the semi-metallic phase becomes unstable to any small amount of disorder. This manifests itself into the instability of the SR fixed point to additional LR disorder: the transition is suppressed and the system always flows towards the strong disordered metallic phase as shown in Fig. 2.

(C) For the opposite case of "short range" disorder correlation, defined by an exponent $a$ larger than a critical value of $a, a>a_{c}(d)$ defined below (39), the SR fixed point is stable and the semimetal to metal SR transition remains unaffected. Fig. 4 shows that the relevant direction for the RG flow is still along $\Delta_{1}$ axis. 


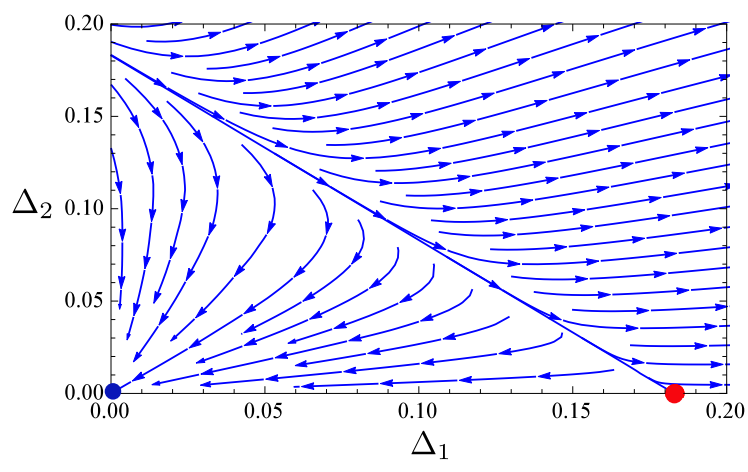

FIG. 4. SR transition: the RG flow for $\mathrm{d}=3(\varepsilon=1)$ and $a=3(\delta=1)$ corresponding to the point C in Fig. 1 1 The red dot is the SR FP with a single unstable direction (relevant operator), describing the semimetal to disordered metal transition governed by the parameter $\Delta_{1}$. The blue dot is the Gaussian FP corresponding to the semimetal phase.

(B) In the intermediate domain $2<a<a_{c}(d)$ we find that in presence of LR disorder a transition still exists between a semi-metal and a disordered metal, but is different from the SR transition : this corresponds to the existence of a new LR fixed point of the RG, see Fig. 3. Correspondingly, the critical properties of this new LR disorder-driven transition are different from the previous SR one.

Thus, we have found that depending on the dimension and the type of algebraic decay, LR correlated disorder can strongly affect the disorder driven transition in semimetals. Indeed, depending on the values of $\varepsilon$ and $\delta$ the RG flow equations 32 and $(33)$ have up to three FPs:

(i) the Gaussian fixed point (Gaussian FP) is

$$
\Delta_{1}^{\mathrm{G}}=\Delta_{2}^{\mathrm{G}}=0
$$

The basin of attraction of this FP in the plane $\left(\Delta_{1}, \Delta_{2}\right)$ corresponds to the semimetal phase. For instance, for $\delta<0$ the basin of attraction collapses to the axe $\Delta_{2}=0$ so that the semimetal phase is washed out by any weak correlated disorder.

(ii) the short-range fixed point (SR FP) reads

$$
\begin{aligned}
& \Delta_{1}^{\mathrm{SR}}=\frac{1}{4}(\sqrt{2 \varepsilon+1}-1)=\frac{\varepsilon}{4}-\frac{\varepsilon^{2}}{8}+O\left(\varepsilon^{3}\right), \\
& \Delta_{2}^{\mathrm{SR}}=0
\end{aligned}
$$

which has a single unstable direction for $\delta>\delta_{\mathrm{c}}(\varepsilon)$, where

$$
\delta_{\mathrm{c}}(\varepsilon)=\frac{1}{2}(\sqrt{1+2 \varepsilon}+\varepsilon-1) \approx \varepsilon-\frac{\varepsilon^{2}}{4}+O\left(\varepsilon^{3}\right),
$$

and fully unstable otherwise. This defines $a_{c}(d)=2+$ $\delta_{c}(d-2)$. In $d=3$ we find $a_{c}(3) \approx 2.8$. (iii) long-range fixed point (LR FP) reads

$$
\begin{aligned}
\Delta_{1}^{\mathrm{LR}}= & \frac{(4+\delta) \sqrt{\delta+1}-3 \delta-4}{2(\varepsilon-\delta)} \approx \frac{\delta^{3}}{16(\varepsilon-\delta)}+\ldots, \\
\Delta_{2}^{\mathrm{LR}}= & \frac{(\varepsilon-2 \delta-4) \sqrt{\delta+1}+4-\varepsilon+4 \delta}{2(\varepsilon-\delta)} \\
& \approx \frac{\delta}{4}-\frac{\delta^{2} \varepsilon}{16(\varepsilon-\delta)}+\ldots
\end{aligned}
$$

The LR FP is physical, i.e. it corresponds to a positive $\tilde{g}(k)$ and has a single unstable direction for $0<\delta<\delta_{\mathrm{c}}(\varepsilon)$.

We now discuss the critical properties at the transition. For $a<2(\delta<0)$ there is a runaway of the RG flow so that the correlated disorder is always relevant if present. In this case there is no semimetal phase and the DOS at zero energy is finite (see Fig. 2). For $2<a<a_{\mathrm{c}}(d)$ $\left(0<\delta<\delta_{\mathrm{c}}(\varepsilon)\right)$ there is a line of phase transitions separating the semimetal and diffusive metal phases with the critical behavior controlled by the LR FP (see Fig. 3). The corresponding critical exponents computed to twoloop order are

$$
\begin{aligned}
& \frac{1}{\nu_{\mathrm{LR}}}=\delta+\frac{\delta^{2}(2 \delta+\varepsilon)}{4 \varepsilon}+O\left(\varepsilon^{3}, \delta^{3}\right), \\
& z_{\mathrm{LR}}=1+\frac{\delta}{2}+O\left(\varepsilon^{3}, \delta^{3}\right), \\
& \eta_{\mathrm{LR}}=-\frac{\delta(2 \varepsilon-\delta)}{8}+O\left(\varepsilon^{3}, \delta^{3}\right) .
\end{aligned}
$$

Note that the two-loop correction to the dynamic critical exponent (44) vanishes. The RG function (34) used to compute this exponent is related to that for the correlation length exponent $\nu_{\text {Ising }}(d=2)$ of the $2 \mathrm{D}$ Ising model with correlated random bond disorder. Scaling arguments allow one to conjecture [27] that at the transition controlled by the LR FP this exponent is exactly $\nu_{\text {Ising }}(d)=2 / a$ in any $2 \leq d<4$. This is in accordance with the recent two-loop order study of diluted 2D Ising model using the $2+\delta$ expansion [26] in which the twoloop corrections to $\nu_{\text {Ising }}(d=2)$ vanish similarly to 44 . On this basis we can also conjecture that the exact value of the dynamic exponent for the semimetal-disordered metal transition controlled by the LR FP is $z_{\mathrm{LR}}=a / 2$.

For $a_{\mathrm{c}}(d)<a\left(\delta_{\mathrm{c}}(\varepsilon)<\delta\right)$ there is also a line of phase transitions separating the semimetal and diffusive metal phases with the critical behavior controlled by the SR FP (see Fig. 4). The critical exponents at the SR FP to two-loop order are

$$
\begin{aligned}
\frac{1}{\nu_{\mathrm{SR}}} & =\varepsilon+\frac{\varepsilon^{2}}{2}+O\left(\varepsilon^{3}\right), \\
z_{\mathrm{SR}} & =1+\frac{\varepsilon}{2}-\frac{\varepsilon^{2}}{8}+O\left(\varepsilon^{3}\right), \\
\eta_{\mathrm{SR}} & =-\frac{\varepsilon^{2}}{8}+O\left(\varepsilon^{3}\right) .
\end{aligned}
$$

One expects that on the borderline between the regions with SR and LR criticality (see Fig. 1) the dynamic critical exponent is a continuous function, i.e we argue that 
$z_{\mathrm{SR}}(\varepsilon)=z_{\mathrm{LR}}\left(\delta_{c}\right)=a_{c} / 2$. Substituting the one loop result (47) one again arrives at Eq. (39).

Note that our derivation of the critical properties based on a $2+\varepsilon$ expansion is valid up to two loop order. Indeed, we have recently showed that the consistent description of the transition with SR disorder beyond two loops involves an infinite number of relevant operators generated by the RG flow. To overcome this obstacle we proposed an alternative way based on a $4-\varepsilon$ expansion 21. However, generalization of this approach to the case of LR disorder is a non trivial task which remains to be done.

\section{CONCLUSIONS}

We have studied the effect of LR disorder correlations on the semimetal - disordered metal transition. We have found that for slowly decaying correlations $a<2$ the LR correlated disorder is always relevant and drives the system to a diffusive phase: the transition is suppressed and the DOS is finite at zero energy for arbitrary weak disorder. Let us note that in particular this result restricts the range of disorder correlations that can be used in simulations (necessary to uncouple the different Weyl cones) to actually observe the SR transition. More surprisingly, we have found an intermediate regime $2<a<a_{c}(\varepsilon)$ where the system undergoes a new transition from semimetal to diffusive metal at finite disorder strength and the criticality is controlled by a LR FP. This change of criticality under addition of LR disorder correlations is a completely new phenomena distinct from what was known for graphene. It would be of great interest to explore the physical consequences of this crossover, e.g. on the behaviour of the DOS at finite energy along the lines of 25$]$. Another interesting direction for future studies would be possible instantons occuring for rare disorder realisations, which are expected to decay algebraically $[5]$.

\section{ACKNOWLEDGMENTS}

We acknowledge support from the French Agence Nationale de la Recherche through Grants No. ANR-12BS04-0007 (SemiTopo).
[1] F. Evers and A. D. Mirlin, Rev. Mod. Phys. 80, 1355 (2008).

[2] S. V. Syzranov and L. Radzihovsky, (2016), arXiv:1609.05694.

[3] S.-Y. Xu, I. Belopolski, N. Alidoust, M. Neupane, G. Bian, C. Zhang, R. Sankar, G. Chang, Z. Yuan, C.C. Lee, S.-M. Huang, H. Zheng, J. Ma, D. S. Sanchez, B. Wang, A. Bansil, F. Chou, P. P. Shibayev, H. Lin, S. Jia, and M. Z. Hasan, Science (2015).

[4] S.-Y. Xu, N. Alidoust, I. Belopolski, Z. Yuan, G. Bian, T.-R. Chang, H. Zheng, V. N. Strocov, D. S. Sanchez, G. Chang, C. Zhang, D. Mou, Y. Wu, L. Huang, C.-C. Lee, S.-M. Huang, B. Wang, A. Bansil, H.-T. Jeng, T. Neupert, A. Kaminski, H. Lin, S. Jia, and M. Zahid Hasan, Nat Phys 11, 748 (2015).

[5] R. Nandkishore, D. A. Huse, and S. L. Sondhi, Phys. Rev. B 89, 245110 (2014).

[6] J. H. Pixley, D. A. Huse, and S. Das Sarma, Phys. Rev. X 6, 021042 (2016).

[7] B. Sbierski, G. Pohl, E. J. Bergholtz, and P. W. Brouwer, Phys. Rev. Lett. 113, 026602 (2014).

[8] B. Roy, V. Juricic, and S. D. Sarma, (2016), arXiv:1603.00017.

[9] E. Fradkin, Phys. Rev. B 33, 3263 (1986).

[10] A. Altland and D. Bagrets, Phys. Rev. Lett. 114, 257201 (2015)

[11] A. Altland and D. Bagrets, Phys. Rev. B 93, 075113 (2016)

[12] S. V. Syzranov, L. Radzihovsky, and V. Gurarie, Phys. Rev. Lett. 114, 166601 (2015).
[13] S. V. Syzranov, V. Gurarie, and L. Radzihovsky, Phys. Rev. B 91, 035133 (2015).

[14] M. Gärttner, S. V. Syzranov, A. M. Rey, V. Gurarie, and L. Radzihovsky, Phys. Rev. B 92, 041406 (2015)

[15] B.Sbierski, E. J. Bergholtz, and P. W. Brouwer, Phys. Rev. B 92, 115145 (2015).

[16] P. Goswami and S. Chakravarty, Phys. Rev. Lett. 107, 196803 (2011).

[17] K. Kobayashi, T. Ohtsuki, K.-I. Imura, and I. F. Herbut, Phys. Rev. Lett. 112, 016402 (2014).

[18] C.-Z. Chen, J. Song, H. Jiang, Q. feng Sun, Z. Wang, and X. Xie, Phys. Rev. Lett. 115, 246603 (2015).

[19] S. V. Syzranov, P. M. Ostrovsky, V. Gurarie, and L. Radzihovsky, Phys. Rev. B 93, 155113 (2016)

[20] B. Roy and S. D. Sarma, Phys. Rev. B 90, 241112(R) (2014).

[21] T. Louvet, D. Carpentier, and A. A. Fedorenko, (2016), arXiv:1605.02009.

[22] J. H. Pixley, P. Goswami, and S. Das Sarma, Phys. Rev. B 93, 085103 (2016)

[23] S. V. Syzranov, V. Gurarie, and L. Radzihovsky, (2016), arXiv:1604.07947.

[24] A. Croy, P. Cain, and M. Schreiber, Eur. Phys. J. B 82, 107 (2011).

[25] A. A. Fedorenko, D. Carpentier, and E. Orignac, Phys. Rev. B 85, 125437 (2012).

[26] M. Dudka, A. A. Fedorenko, V. Blavatska, and Y. Holovatch, Phys. Rev. B 93, 224422 (2016).

[27] A. Weinrib and B. I. Halperin, Phys. Rev. B 27, 413 (1983) 